\section{Dr. Carter, et al reply}

\section{To the Editor:}

We thank Winger and Reed for their comments regarding our article ${ }^{1}$.

As with any study, ours had limitations. Winger and Reed point out that the data collected are provided voluntarily and, therefore, may not represent the true number of congenital anomalies within the population. We agree. These data represent a "best-case scenario." It is impossible for there to be fewer congenital anomalies than those actually reported. As we know from any postmarketing data, significant under-reporting is the norm. They also suggest that the data reviewed did not support the calculation of a denominator necessary for the calculation of incidence. It is true that the total number of pregnant women treated with a tumor necrosis factor (TNF) antagonist during pregnancy is an unknown; this was acknowledged in the original article. However, we did not attempt to calculate an incidence of the reported birth defects in the article. The "incidences" listed in Table 2 are historical controls. Winger and Reed also raise the issue of concomitant medications as a potential teratogen. As stated in the article, the majority (59\%) of these women were taking either etanercept or infliximab monotherapy. Of the minority of patients taking concomitant therapies, 5 were on methotrexate and 2 were on leflunomide. There was no trend in observed anomalies in women taking concomitant medications.

Winger and Reed suggest that women who continue TNF antagonists into pregnancy are likely to have more active disease that might be associated with adverse outcome independent of the medication taken. We would argue the opposite. Anti-TNF therapy has a dramatic effect on disease activity in a large percentage of patients who take these drugs. It would seem logical that only women who demonstrate dramatic improvement while on a TNF antagonist would consider continuing therapy into pregnancy. This would create a selection bias of decreased background disease activity in those women willing to continue the drug.

Another contention was that the congenital anomalies reported tend to be the most common in the population, potentially compromising correlation to the drug. As a reminder, we considered a $\mathrm{p}$ value to be significant only if less than 0.001 . Using this very strict definition of significance, $21 \%$ of the anomalies occurred significantly more frequently than in historical controls.

Winger and Reed reiterate that VACTERL association is a constellation of 7 anomalies, with 3 required for a diagnosis. They incorrectly state that no patient reported in this study qualifies for the diagnosis. Subject 19 (Table 1) has VACTERL. This subject was intentionally removed from the data analysis to eliminate statistical bias. We are also personally aware of a second child born with VACTERL to a mother taking adalimumab (reported after the 2005 data cutoff for this study). Unfortunately, this child passed away at the age of 8 months secondary to complications from her birth defects. We would like to stress that our hypothesis is not that these drugs cause VACTERL, rather that they cause the anomalies that encompass this non-random association of birth defects.

Finally Winger and Reed suggest that we used the US Food and Drug Administration (FDA) data in a "prohibited manner." First, we obtained these data through the Freedom of Information Act, not through the Adverse Event Reporting System (AERS) website as they suggest (the Webpage they referenced also does not state those prohibitions). More importantly, we did not attempt to "calculate incidence" in our study. We stated the number of reports and calculated Poisson probabilities. Also, nowhere in the article did we attempt to "estimate drug risk;" we simply compared the reported numbers to data of historical controls. Finally, we made no attempt at "comparisons between drugs." Data from all TNF antagonists were requested, and the data were viewed en bloc. We did not compare individual TNF antagonists to each other or to other drugs or drug classes.

Debate is a healthy process in the absence of conclusive data. As stated in our original article, these data are not definitive, but suggest possible causation. Regardless of causation, 61 congenital anomalies in 41 children born to mothers taking TNF antagonists have been reported to the FDA through 2005 and there have been more reports since that time. As a comparison, mycophenolate mofetil was recently changed from a pregnancy category $\mathrm{C}$ to $\mathrm{D}$ after post-marketing reports revealed 18 children with anomalies $^{2}$. The package inserts of all the TNF antagonists argue against the use of these drugs during pregnancy, suggesting they be utilized only if there is a "clear need."

We find it interesting that Winger and Reed have studied anti-TNF therapy with and without intravenous immunoglobulin (IVIG) as a potential treatment for female infertility ${ }^{3}$. This retrospective analysis of clinical data demonstrates that adalimumab does not significantly improve outcomes compared to controls; it also offers no significant extra benefit when combined with IVIG compared to IVIG alone. Such experimentation with antiTNF therapy is warranted in the setting of a clinical trial that utilizes a full informed consent process. Any similar off-label treatment as part of routine clinical care would be cavalier.

JOHN D. CARTER, MD, Associate Professor of Medicine; LOUIS R. RICCA, MD; JOANNE VALERIANO, MD; FRANK B. VASEY, MD, Department of Internal Medicine, Division of Rheumatology, University of South Florida College of Medicine, 12901 Bruce B. Downs Blvd., MDC 81, Tampa, Florida, USA. Address correspondence to Dr. Carter; E-mail: jocarter@health.usf.edu

\section{REFERENCES}

1. Carter JD, Ladhani A, Ricca LR, Valeriano J, Vasey FB. A safety assessment of tumor necrosis factor antagonists during pregnancy: A review of the Food and Drug Administration database. J Rheumatol 2009;36:635-41.

2. Important changes in the CellCept (mycophenolate mofetil) prescribing information. Use of CellCept is associated with recurrent pregnancy loss and congenital malformations/ change from pregnancy category $\mathrm{C}$ to pregnancy category D. [Internet. Accessed April 22, 2009.] Available from: http://www.fda.gov/ medwAtch/SAFETY/2007/CellCept_dearhcpoct07.pdf

3. Winger EE, Reed JL, Ashoush S, Ahuja S, El-Toukhy T, Taranissi M. Treatment with adalimumab (Humira ${ }^{\circledR}$ ) and intravenous immunoglobulin improves pregnancy rates in women undergoing IVF. Am J Reprod Immunol 2009;61:113-20.

J Rheumatol 2009;36:9; doi:10.3899/jrheum.090283 